Artículo original

\title{
APROVECHAMIENTO DE LAS CABEzAS DEL DESTILADO DE PISCO DE UVA NEGRA CRIOLLA MEDIANTE REDESTILACIÓN PARA LA ELABORACIÓN DE ANISADO
}

USE OF PISCO DISTILLATE HEADS FROM CRIOLLO BLACK GRAPE DISTILLATE BY REDISTILLATION FOR THE PRODUCTION OF ANISETTE

\author{
EnRIQue Alexander Concha Pereira ${ }^{1}$ \\ (D) https://orcid.org/0000-0003-1941-4804 \\ TOMÁs DELGado CABRERA ${ }^{2}$ \\ (D) https://orcid.org/0000-0002-2890-3661
}

Información del artículo: Recibido: 21/01/2021 Aceptado: 01/06/2021 Publicado: 28/06/2021

${ }^{1}$ Escuela de Ingeniería Agroindustrial, Universidad Privada de Tacna ${ }^{2}$ Docente en la Escuela de Ingeniería Agroindustrial, Universidad Privada de Tacna E-mail: ${ }^{1}$ alexconchapereira@hotmail.com, ${ }^{2}$ tdelgadoc62@gmail.com 


\section{Resumen}

Se aprovechó las fracciones de cabeza del destilado de pisco de uva Negra Criolla, mediante redestilación, para la elaboración de anisado. Evaluando la influencia del grado alcohólico de las fracciones de cabeza y la concentración de anís en el rendimiento, así como las características fisicoquímicas y aceptabilidad del anisado. Mediante un diseño experimental y, un panel de catadores entrenados y una ficha de cata hedónica. Para la cantidad de se estudió el efecto de tres componentes en 7 corridas. El diseño se ejecutó en un solo bloque. El orden de los experimentos fue aleatorio. Para la optimización de la elaboración del destilado se empleó el método de la función deseada. Las fracciones de cabeza de la destilación del pisco de uva Negra Criolla presentaron un grado alcohólico de $61^{\circ} \mathrm{GL}$ y una densidad de 1,5228. Las siete muestras analizadas, presentaron una relación estadísticamente significativa entre la densidad y el volumen, densidad y rendimiento, así como densidad y análisis sensorial del anisado, con un nivel de confianza del 95,0 \%. Así mismo existe una relación estadísticamente significativa entre el rendimiento y el análisis sensorial. La aceptabilidad analizada da como resultado que la muestra 5 con proporción 20:79:01 (cabeza, agua y anís), presenta la mayor aceptación, con valoración de 6,54 en una escala hedónica de 1 a 9 . Así se concluye que se logró aprovechar las fracciones de cabeza del destilado de pisco de uva Negra Criolla mediante redestilación para la elaboración de anisado.

Palabras Clave: Anisado, Pisco, Cabeza, Redestilación.

\section{Abstract}

The head fractions of Negra Criolla grape pisco distillate were used, by redistillation, for the production of anisette. The influence of the alcohol content of the head fractions and the concentration of aniseed on the yield, as well as the physicochemical characteristics and acceptability of the anisette was evaluated. By means of an experimental design, a panel of trained tasters and a hedonic tasting sheet. For the quantity of aniseed, the effect of three components was studied in 7 runs. The design was executed in a single block. The order of the experiments was randomised. For the optimisation of the distillate production, the desired function method was used. The head fractions from the distillation of Negra Criolla grape pisco had an alcohol content of $61^{\circ} \mathrm{GL}$ and a density of 1.5228 . The seven samples analysed showed a statistically significant relationship between density and volume, density and yield, as well as density and sensory analysis of aniseed, with a confidence level of $95.0 \%$. There is also a statistically significant relationship between yield and sensory analysis. Acceptability analysis showed that sample 5 with a ratio of 20:79:01 (head, water and aniseed) was the most acceptable, with a rating of 6.54 on a hedonic scale of 1 to 9 . It can be concluded that the head fractions of the Negra Criolla grape pisco distillate were used by redistillation for the production of anisette.

Keywords: Aniseed, Pisco, Head, Redistillation. 


\section{Introducción}

La semilla de anís era considerada, por egipcios, griegos y romanos, un remedio eficaz contra afecciones gastrointestinales. El nacimiento del anís (anisado) no es del todo claro. Unos lo sitúan en Provenza y Aquitania, otros en tiempos de Adberramán III, gran aficionado a los alambiques y convencido de las virtudes del anís. Pero lo que parece más probable es que fueran los holandeses, que tenían la exclusividad en el comercio de la badiana (anís estrellado o de China)y eran grandes maestros en la destilación del vino, los que extendieron el gusto por este tipo de aguardiente (Jurado, 2004).

El mercado actual exige contar con empresas pisqueras competitivas, más allá de las ventajas comparativas que las posicionan tímidamente, es el agregar valor y reducir costos lo que las impulsa a horizontes más prometedores, en este sentido es importante aprovechar las fracciones de cabeza del destilado del pisco, para la producción de aguardientes que diversifiquen la oferta de las empresas en dmercado, generando ingresos y aumentando la rentabilidad del sector.

La presente investigación, consiste en el aprovechamiento de las fracciones de cabeza del destilado de pisco de uva Negra Criolla mediante redestilación parala elaboración de anisado. Actualmente la región de Tacna cuenta con 1,680 empresas formales instaladas que operan activamente, de las mismas que sólo el $21,07 \%$ se dedican a la Agroindustria, de estas 358 empresas, las vitivinícolas son 52 empresas que representa el 14,53 \% y las empresas de destilación y mezcla debebidas alcohólicas identificadas como productoras de pisco, que son 18 con el4,47\%, entre otros productores. (Gobierno Regional de Tacna, 2009); las empresas pisqueras, creadas durante los últimos años, pueden perder ingresos al no optimizar sus recursos, perdiéndose la oportunidad de ganar competitividad y desarrollar la agroindustria en la región.

Puede mejorarse este panorama, brindando al productor pisquero nuevas herramientas tecnológicas que mejoren sus ingresos, a través de la recuperación de los alcoholes perdidos en los sub productos de la destilación, controlando las concentraciones de alcohol etílico de cada sub producto y a través de un proceso de redestilación, obteniendo un destilado que puede usarse en producción de anisados.

¿Se podría aprovechar las fracciones de cabeza del destilado de pisco de uva Negra Criolla mediante redestilación para la elaboración de anisado? El estudio permitirá evaluar la influencia del grado alcohólico de las fracciones de cabeza y la concentración de anís en la destilación de las fracciones de cabeza del pisco de uva negra criolla, en las características fisicoquímicas y sensoriales del anisado. El estudio ayudaría a los productores de pisco a mejorar su competitividad al diversificar su oferta de licores al obtener anisado de buena calidad y aceptabilidad. La mejora en la competitividad de las empresas pisqueras contribuirá a que los productores de pisco tengan mayores ventas e ingresos.

Hatta (2004) estudió la influencia de la fermentación con orujos en los componentes volátiles del pisco de uva Italia (Vitis vinífera L. var. Italia), determinando la evolución de los componentes volátiles del pisco durante la destilación, para lo cual elaboraron un pisco con Uva Italia, de acuerdo al método tradicional, separando el destilado en 17 fracciones $(F n)$ con volúmenes de: F1 y F2: 50 ml, F3 a F13: $200 \mathrm{ml}$ y F14 a F17: $100 \mathrm{ml}$ y en cada fracción cuantificaron los compuestos mayoritarios volátiles considerados en la NTP 211.001. Como resultado se obtuvo que en conjunto las dos fracciones de cabeza presentaron $64,2 \circ$ GL y expresado en $\mathrm{ml} / 100 \mathrm{~mL}$ aa, 156,53 de ésteres (formiato de etilo 0.50, acetato de etilo y acetato de isoamilo 12,09), 24,61 de acetaldehído, 722,99 de alcoholes superiores (91,27 de isobutanol, 551,45 de isoteramilico, 1,28 de butanol y 79,00 de propanol, no registrándose isopropanol), 46,77 de metanol, 1,58 de ácido acético, no registrándose furfural en esta fracción.

Garrido, Linares y Cárdenas (2008) buscaron conocer la composición de estas fracciones y la evolución de la composición de las fracciones a lo largo del proceso de destilación, para lo cual destilaron mostos de uva Torontel y Quebranta, el resultado obtenido mostró en $\mathrm{mg} / 100 \mathrm{~mL}$, para el mosto de Quebranta, que los componentes descendían del inicio al final de la destilación en ésteres de 
97,17 a 1,39, acetaldehído de 6,55 a 2,06, alcoholes superiores de 442,21 a 24,53 y metanol de 11,94 a 8,69. Del mismo modo para el mosto de Torontel, en ésteres de 135,27 a 1,72, acetaldehído de 4,44 a 2,70, alcoholes superiores de 205,79 a 7,77 y metanol de 12,21 a 5,43.

Rota y Faria (2009), evaluaron el efecto del proceso de bidestilación en lacalidad sensorial de la Cachaza, realizando las destilaciones sucesivas de caldos fermentados de caña, en alambiques de cobre y acero inoxidable, así se demostró que el proceso de bidestilación en alambique de cobre da como resultado mejores efectos positivos en la aceptación en relación al sabor, impresión global, aroma alcohólico, sabor de alcohol y saborresidual, que las muestras de una sola destilación y en alambique de acero inoxidable.

Karapanagioti y Bekatorou (2014), estudiaron las características de la dilución del anisado Ouzo con agua destilada, correlacionado con la conductividad de la dilución. Las bebidas auténticas demostraron una baja conductividad, lo que sugiere el uso de agua tratada, mientras que las bebidas de fraude se mezclaron con agua potable. Dambergs, Kambouris, Francis y Gishen (2002), estudiaron un método de análisis rápido del metanol en la destilación de derivados de la uva, por cromatografía de gases (GC) y espectroscopia de infrarrojo cercano (NIRS). La mejor exactitudde los modelos de predicción, medida por el error estándar de valores de predicción, era 0,06 g / L de metanol.

\section{Objetivos}

Aprovechar las fracciones de cabeza del destilado de pisco de uva NegraCriolla mediante redestilación para la elaboración de anisado.

\section{Metodología}

a) Variables Independientes: Cabeza (\% Vol/Vol) y Anís (\% $\%$ ol/Vol).

Tabla 1

Variables Independientes.

\begin{tabular}{lccc}
\hline Variables & \multicolumn{3}{c}{ Niveles } \\
& Mínimo & Intermedio & Máximo \\
& -1 & 0 & +1 \\
\hline Cabeza (\% en la mezcla) & 50 & 30 & 10 \\
Anís (\% en lamezcla) & 1 & 5 & 10 \\
\hline
\end{tabular}

b) Variables Dependientes: Rendimiento, Anisado, Análisis fisicoquímico y Aceptabilidad. Indicadores de las variables dependientes:

- Grado alcohólico: Según Norma Técnica Peruana

- Densidad picnométrica: Según Norma Técnica Peruana

- Características sensoriales: Aspecto general, color, olor, sabor, equilibrio e intensidad aromática en una escala hedónica de 1 a 9 puntos.

Se utilizó un diseño de investigación de tipo experimental para determinarel efecto de los dos factores (mezclas de cabeza y anís) sobre las características fisicoquímicas y sensoriales del anisado, el diseño de investigación aplicado, destacando a los factores o variables en estudio.

La descripción de la metodología seguida para la elaboración del anisado es como sigue:

- Recepción de materia prima: Fracciones de cabeza de destilación de pisco de uva Negra Criolla a la cual se le analizará el grado alcohólico. 
- Destilado: Se debe realizar una destilación directa y discontinua, proceso quese lleva a cabo empleando un alambique simple de cobre, en la cual se llenará con la porción de cabeza, agua y anís según el diseño estadístico.

- Análisis: A las muestras obtenidas se les realiza los análisis fisicoquímicos (determinación del contenido de Grado alcohólico y Densidad Picnométrica) y evaluación sensorial planteada para con estos datos obtenidos proceder al análisis estadístico final.

La muestra de estudio se obtuvo de la Bodega "Don Miguel" del distrito de Pocollay de la Provincia de Tacna, la cual consta de 20 litros de cabeza de destilación de pisco de Negra Criolla a la vendimia 2015 a esta materia prima se le realizó un análisis fisicoquímico, donde se determinó los ${ }^{\circ} \mathrm{GL}$ mediante un alcoholímetro.

Técnicas e instrumentos para recolección de datos

- Análisis Fisicoquímicos: Determinación del contenido de alcohol etílico: Mediante medición directa con alcoholímetro.

- Análisis sensorial: Se realizó un análisis sensorial mediante un panel de catadores entrenados y conformados por personas muy bien informadas acerca del modo operativo de análisis y productores de piscos con una ficha de cata de preferencia con escala estructurada de 1 a 9 puntos.

- Evaluando: Vista, Olfato, Gusto, y Armonía

Análisis de datos: Para la cantidad de variables independientes elegidas se utilizó el diseño experimental, de vértices extremos el cual estudió el efecto de tres componentes en 7 corridas. El diseño se ejecutó en un solo bloque. El orden de los experimentos fue completamente aleatorizado.

En la tabla 2 se muestra la distribución de las correspondientes mezclas del diseñó experimental elegido.

Tabla 2 Delineamiento experimental

\begin{tabular}{cccc}
\hline mezcla & fracciones de cabeza (I) & agua (l) & anís (kg) \\
\hline 1 & 1,00 & 3,95 & 0,05 \\
2 & 2,45 & 2,50 & 0,05 \\
3 & 2,00 & 2,50 & 0,50 \\
4 & 1,00 & 3,50 & 0,50 \\
5 & 1,00 & 3,95 & 0,05 \\
6 & 2,45 & 2,50 & 0,05 \\
7 & 2,00 & 2,50 & 0,50
\end{tabular}

Con los datos de los 07 tratamientos para las variables respuestas se desarrolló para cada variable respuesta, modelos matemáticos de primer orden conteniendo los términos lineales, cuadráticos y de interacción; y para determinar sus coeficientes, se empleó la metodología de superficie de respuesta. El modelo para ser considerado predictivo en la región analizada debe presentar regresión significativa $(P<0,05)$, falta de ajuste no significativo en el mismo nivel de significancia y alto valor $R$ (más próximo de 1). Para la optimización de la elaboración del destilado consiste en estandarizar cada respuesta en una función $\mathrm{Fd}$ cuyo valor varía de 0 (fuera del rango deseado) a 1 (en el rango deseado).

La presente investigación, análisis fisicoquímico y sensorial se realizó en los laboratorios de la Escuela Profesional de Ingeniería Agroindustrial de la Universidad Privada de Tacna. Se utilizó un diseño experimental, de Vértices Extremos el cual estudiará el efecto de tres componentes en 7 corridas. Los reactivos que se emplearon en los diferentes métodos de esta investigación fueron de pureza analítica.

El desarrollo del proceso de destilación para evaluar las proporciones de más adecuadas para el aprovechamiento de las fracciones de cabeza de destilación en la elaboración de anisado obedecerá al flujo grama representado en la figura 1. 
Figura 1. Etapas de la investigación

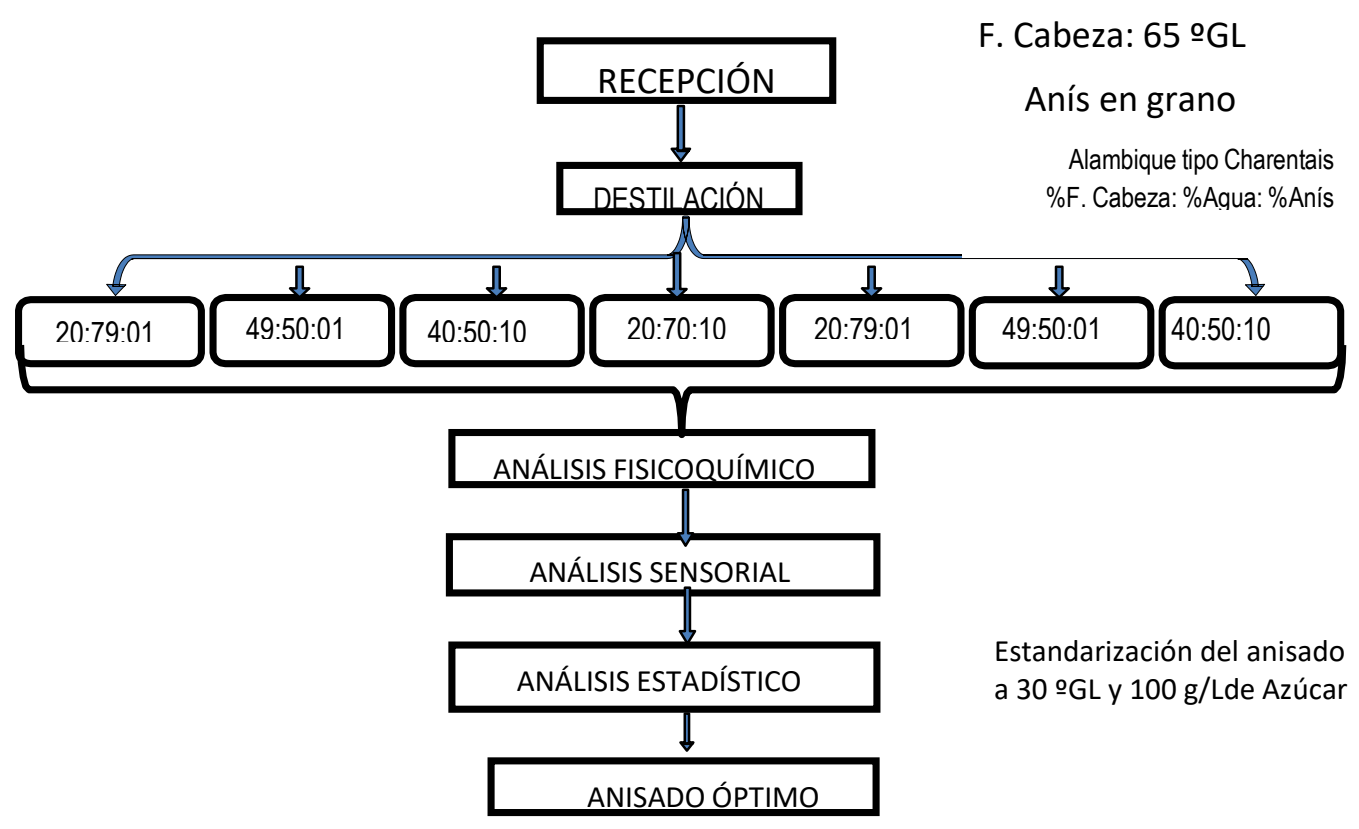

Técnicas e instrumentos para la recolección de datosAcciones y actividades:

Objetivo 1: Analizar las fracciones de cabeza de pisco de uva NegraCriolla.

- La parte experimental de la investigación en una primera etapa se analizó la muestra de cabeza de las destilaciones de pisco de uva Negra Criolla, mediante la medición del grado alcohólico por lectura directa y la determinación de su densidad por picnometría.

- Determinación del grado alcohólico de la cabeza de destilado fue con un alcoholímetro por lectura directa.

- Determinación de la densidad por picnometría. Fue con un picnómetro y balanza analítica.

Objetivo 2: Analizar fisicoquímicamente las muestras de anisado.

A las muestras obtenidas se les determino el grado alcohólico y densidad.

Objetivo3: Evaluar la aceptabilidad de las muestras de anisado elaboradas con fracciones de cabeza de destilado de pisco de uva Negra Criolla.

\section{Test de aceptación - preferencia}

Este test tiene como objetivo medir actitudes subjetivas comoaceptación o preferencia de las muestras de anisados entre sí, mediante una escala hedónica en jueces no entrenados, se utiliza fichas elaboradas para tal fin y las muestras estandarizadas a $30 \mathrm{GL}$ y $100 \mathrm{~g} / \mathrm{L}$ de Azucares totales, son presentadassimultáneamente en orden aleatorio.

Se utilizó el programa statgraphics centurión, para crear un diseño devértices extremos, determinando si existe diferencia significativa entre las variables a un 95 y $99 \%$ de confiabilidad.

\section{Resultados}

Análisis fisicoquímico: Se aprovechó las fracciones de cabeza del destilado de pisco de uva Negra Criolla mediante redestilación para la elaboración de anisado, analizando fisicoquímicamente las muestras de anisado, en el laboratorio y reportaron los resultados expresados en la tabla 3. Los grados alcohólicos de dos de las siete muestras, se encuentran dentro del tipo de anisados secos y extrasecos, los cinco restantes se encuentran por encima de dichas características (Jurado, 2004). Sin embargo, para fines de evaluación sensorial las muestras fueron estandarizadas a $30^{\circ} \mathrm{GL}$ y $100 \mathrm{~g} / \mathrm{L}$ de azucares totales, por lo que, según la Norma Técnica Peruana, serían evaluados como anisados semi secos. 


\section{Tabla 3}

Determinación de la densidad del anisado por picnometría.

\begin{tabular}{ccc}
\hline Muestra & Densidad & ${ }^{\circ} \mathrm{GL}$ \\
\hline Cabeza & 1,5228 & 61 \\
Muestra 1 & 1,5489 & 50 \\
Muestra 2 & 1,5354 & 57 \\
Muestra 3 & 1,5164 & 64 \\
Muestra 4 & 1,5336 & 57 \\
Muestra 5 & 1,5515 & 48 \\
Muestra 6 & 1,5374 & 56 \\
Muestra 7 & 1,5185 & 65 \\
\hline
\end{tabular}

Fuente: Elaboración propia.

\section{Tabla 4}

Relación de la densidad de las muestras de anisado y el volumendestilado. ANOVA

\begin{tabular}{|c|c|c|c|c|c|}
\hline Fuente & $\begin{array}{l}\text { Suma } \\
\text { Cuadrados }\end{array}$ & Gl & $\begin{array}{l}\text { Cuadrado } \\
\text { Medio }\end{array}$ & $\begin{array}{l}\text { Razón- } \\
F\end{array}$ & Valor-P \\
\hline Modelo Lineal & 0,00106014 & 2 & 0,000530071 & 71,02 & 0,0008 \\
\hline Error & 0,000029853 & 4 & 0,000007463 & & \\
\hline total & 1 & & 29 & & \\
\hline Total (corr.) & 0,00108999 & 6 & & & \\
\hline
\end{tabular}

Fuente: Elaboración propia.

En la tabla 4, muestra un análisis de varianza para el modelo lineal. Dado que el valor-P para este modelo es menor que 0.05 , existe una relación estadísticamente significativa entre la densidad y el volumen destilado de las muestras de anisado, con un nivel de confianza del $95.0 \%$.

En la tabla 5 se muestra la relación de la densidad de las muestras de anisado y el rendimiento del destilado fueron sometidas a evaluación estadística, con el siguiente resultado:

Tabla 5. Relación de la densidad de las muestras de anisado y el rendimiento deldestilado.

\section{ANOVA}

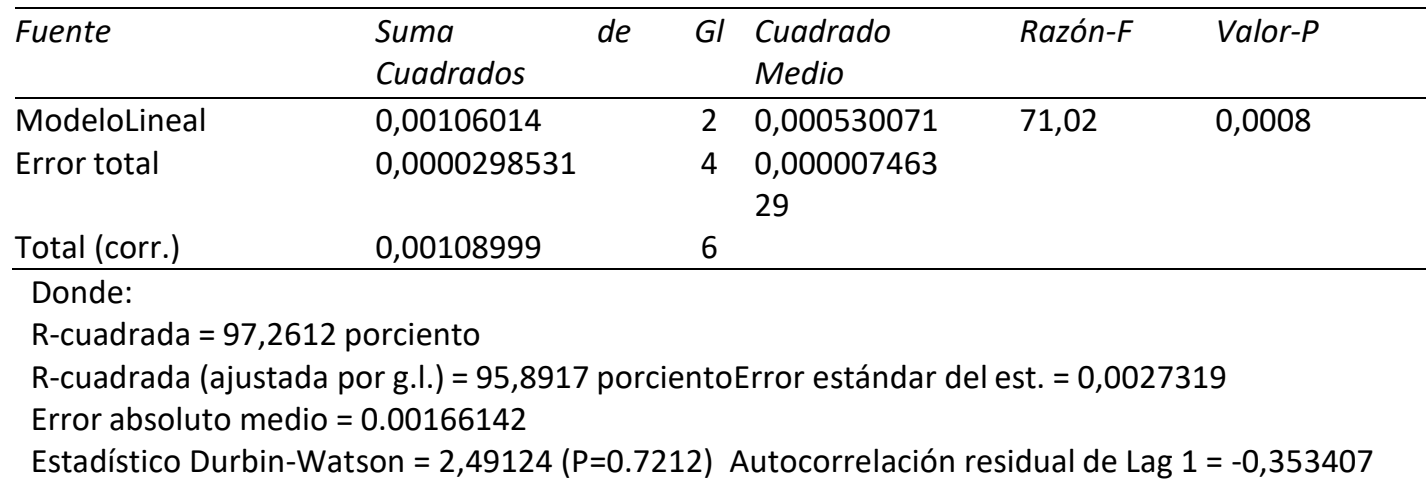

Esta tabla 5, se muestra un análisis de varianza y dado a que el valor-P paraeste modelo es menor que 0.05 , existe una relación estadísticamente significativa entre la Densidad y el Rendimiento, con un nivel de confianza del $95.0 \%$.

Se evaluó la aceptabilidad de las muestras de anisado elaboradas con fracciones de cabeza de destilado de pisco de uva Negra Criolla, como se aprecia en la tabla 6, en donde se muestra la relación 
de la densidad de las muestras de anisado y el análisis sensorial del destilado fueron sometidas a evaluación estadística, con el siguiente resultado:

\section{Tabla 6}

Relación de la densidad de las muestras de anisado y el análisissensorial del destilado.

\section{ANOVA}

\begin{tabular}{|c|c|c|c|c|c|}
\hline Fuente & Suma de Cuadrados & Gl & $\begin{array}{c}\text { Cuadrado } \\
\text { Medio }\end{array}$ & Razón-F & Valor-P \\
\hline $\begin{array}{l}\text { Modelo } \\
\text { Lineal }\end{array}$ & 0,00106014 & 2 & 0,000530071 & 71,02 & 0,0008 \\
\hline Error total & 0,0000298531 & 4 & 0,00000746329 & & \\
\hline Total (corr.) & 0,00108999 & 6 & & & \\
\hline $\begin{array}{l}\text { Donde: } \\
\text { R-cuadrada = 97, } \\
\text { R-cuadrada (ajus } \\
\text { Error absoluto m }\end{array}$ & $\begin{array}{l}12 \text { porciento } \\
\text { da por g.l. }=95.8917 \text { porcie } \\
\text { lio }=0,00166142\end{array}$ & toEr & ror estándar del est. & 0027319 & \\
\hline Estadístico Durbi & Watson $=2,49124(P=0,72$ & & & & \\
\hline
\end{tabular}

La tabla 6, muestra un análisis de varianza para el modelo linealentre la densidad y el análisis sensorial. Dado que el valor-P para este modelo es menor que 0.05 , existe una relación estadísticamente significativaentre la densidad y el análisis sensorial, con un nivel de confianza del $95,0 \%$.

\section{Análisis de Rendimiento}

En el proceso de destilación se pudo determinar el rendimiento del alcohol recuperado de la destilación de las fracciones de cabeza para su posterior producción de destilado, estos datos se tienen determinados en la tabla 8.

Tabla 8. Determinación del rendimiento de alcohol recuperado en la destilación delas fracciones de cabeza para la producción de anisado.

\begin{tabular}{cccccc}
\hline $\begin{array}{c}\mathrm{N}^{\circ} \text { Cabeza } \\
\text { (Litros) }\end{array}$ & Alcohol(Litros) & Anisado(Litros) & Alcohol(Litros) & Rendimiento(\%) \\
\hline 1 & 1,0000 & 0,6100 & 1,0000 & 0,50 & 81,9672 \\
2 & 2,4500 & 1,4945 & 2,3830 & 0,57 & 90,8873 \\
3 & 2,0000 & 1,2200 & 1,6000 & 0,64 & 83,9344 \\
4 & 1,0000 & 0,6100 & 0,6850 & 0,57 & 64,0082 \\
5 & 1,0000 & 0,6100 & 1,0000 & 0,48 & 78,6885 \\
6 & 2,4500 & 1,4945 & 2,4130 & 0,56 & 90,4169 \\
7 & 2,0000 & 1,2200 & 1,6000 & 0,65 & 85,2459 \\
\hline
\end{tabular}

El rendimiento se vio afectado en las muestras 1, 3, 4, 5 y 7 por la presencia de destilados blanquecinos hacia el final de la destilación, proporción que no fue unida al cuerpo del destilado, reduciendo su volumen final y grado alcohólico.

En la tabla 9, se tiene las fracciones de destilado tanto en grado alcohólico como volumen de la fracción separada. 
Tabla 9

Fracciones de destilado del anisado.

\begin{tabular}{ccccccc}
\hline $\mathrm{N}^{\circ}$ & Cabeza $(\mathrm{mL})$ & ${ }^{\circ} \mathrm{GL}$ & Cuerpo $(\mathrm{mL})$ & ${ }^{\circ} \mathrm{GL}$ & Cola $(\mathrm{mL})$ & ${ }^{\circ} \mathrm{GL}$ \\
\hline 1 & 100 & 68 & 1000 & 50 & 400 & 17 \\
2 & 250 & 79 & 2383 & 57 & - & - \\
3 & 200 & 78 & 1600 & 64 & 600 & 12 \\
4 & 100 & 67 & 685 & 57 & 830 & 21 \\
5 & 100 & 65 & 1000 & 48 & 500 & 14 \\
6 & 245 & 79 & 2413 & 56 & - & - \\
7 & 200 & 78 & 1600 & 65 & 600 & 11 \\
\hline
\end{tabular}

En la tabla 10, se tiene la relación del rendimiento de las muestras de anisado y el análisis sensorial del destilado fueron sometidas a evaluación estadística, con el siguiente resultado:

Tabla 10. Relación del rendimiento de las muestras de anisado y el análisissensorial del destilado para Rendimiento.

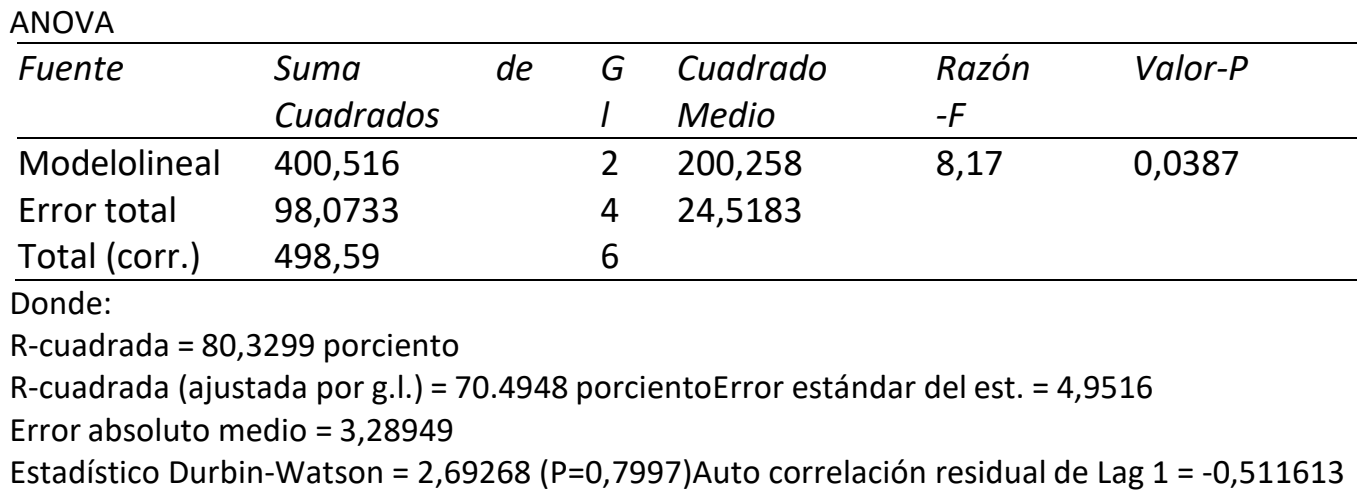

En la tabla 10, se muestra un análisis de varianza para el modelolineal actualmente seleccionado. Dado que el valor-P para este modelo es menor que 0.05 , existe una relación estadísticamente significativa entre rendimiento y análisis sensorial, con un nivel de confianza del $95.0 \%$. El estadístico R-Cuadrada indica que el modelo, así ajustado, explica 80,3299 \% de la variabilidad en Rendimiento. EI estadístico R-cuadrada ajustada, que es más adecuado para comparar modelos con diferente número de variables independientes, es 70,4948 \%. El error estándar del estimado muestra que la desviación estándar de los residuos es 4,9516. El error medio absoluto (MAE) de 3.28949 es el valor promedio de los residuos.El estadístico de Durbin-Watson (DW) prueba los residuos para determinarsi haya alguna correlación significativa basada en el orden en que se presentan los datos en el archivo. Puesto que el valor-P es mayor que 5,0\%, no hay indicación de auto correlación serial en los residuos con un nivel de significancia del $5,0 \%$.

La ecuación del modelo ajustado es Rendimiento $=93.0017 *$ Cabeza $+77.9782 *$ Agua $+54.9197 *$ Anís

\subsection{Aceptabilidad}

En la tabla 11 se tiene los resultados obtenidos del análisis de varianza parala aceptabilidad de las muestras de anisado. 
Tabla 11

Análisis de Varianza para Resultado - Suma de Cuadrados Tipo III. ANOVA

\begin{tabular}{lcclll}
\hline Fuente & $\begin{array}{c}\text { Suma de } \\
\text { Cuadrados }\end{array}$ & Gl & $\begin{array}{l}\text { Cuadrado } \\
\text { Medio }\end{array}$ & Razón-F & Valor-P \\
\hline Efectos Principales & & & & & \\
A:Jueces & 33,8724 & 6 & 5,64541 & 3,98 & 0,0037 \\
B:Muestras & 42,4796 & 6 & 7,07993 & 5,00 & 0,0008 \\
Residuos & 51,0204 & 36 & 1,41723 & & \\
Total (Corregido) & 127,372 & 48 & & & \\
\hline
\end{tabular}

Todas las razones- $\mathrm{F}$ se basan en el cuadrado medio del error residual

La tabla 11, ANOVA descompone la variabilidad de resultado en contribuciones debidas a varios factores. Puesto que se ha escogido la suma de cuadrados Tipo III (por omisión), la contribución de cada factor se mide eliminando los efectos de los demás factores. Los valores-P prueban la significancia estadística de cada uno de los factores. Puesto que 2 valores-P son menores que 0.05 , estos factores tienen un efecto estadísticamente significativo sobre Resultado con un $95.0 \%$ de nivel de confianza.

El tests de aceptación - preferencia, habría producido reacciones subjetivas en el consumidor, sirviéndoles para preferir aquellos que presentaba mejores descriptores aromáticos característicos, para (Tsachaki, 2010) estos serían: el anís, el dulce, alcohólico, herbáceo, vainilla y mentol; sabores dulces, alcohólicos, picantes, artificiales, aromáticos, mentolados o cáusticos; y retrogustos dulces, alcohólicos, artificiales, picantes y amargos. Sin embargo, para la Norma Técnica Peruana, son requisitos únicamente el aspecto incoloro, cristalino y aroma a anís, libre de sabores extraños, por lo que los destilados obtenidos simplemente cumplen con tal normativa.

En la tabla 12 se presenta los resultados de la prueba de rangos paralos resultados finales de las muestras.

\section{Tabla 12}

Pruebas de Múltiple Rangos para Resultado por MuestrasMétodo: 95.0 porcentaje LSD

\begin{tabular}{lllll}
\hline Muestras & Casos & Media LS & Sigma LS & $\begin{array}{l}\text { Grupos } \\
\text { Homogéneos }\end{array}$ \\
\hline 4 & 7 & 4,25 & 0,537356 & $\mathrm{X}$ \\
7 & 7 & 4,28571 & 0,537356 & $\mathrm{X}$ \\
3 & 7 & 4,78571 & 0,537356 & $\mathrm{XX}$ \\
6 & 7 & 4,78571 & 0,537356 & $\mathrm{XX}$ \\
1 & 7 & 5,96429 & 0,537356 & $\mathrm{XX}$ \\
2 & 7 & 6,5 & 0,537356 & $\mathrm{X}$ \\
5 & 7 & 6,53571 & 0,537356 & $\mathrm{X}$ \\
\hline
\end{tabular}

En la figura 6, se presenta la gráfica de cigote, donde se puede ver las de mayor puntuación y las de menor puntuación, dentro de las primeraslas de mayor aceptación se encuentran las muestras 2 y 5. 


\section{Figura 6}

Representación de muestras por su aceptación.

Medias y $\mathbf{9 5 . 0 \%}$ de Fisher LSD

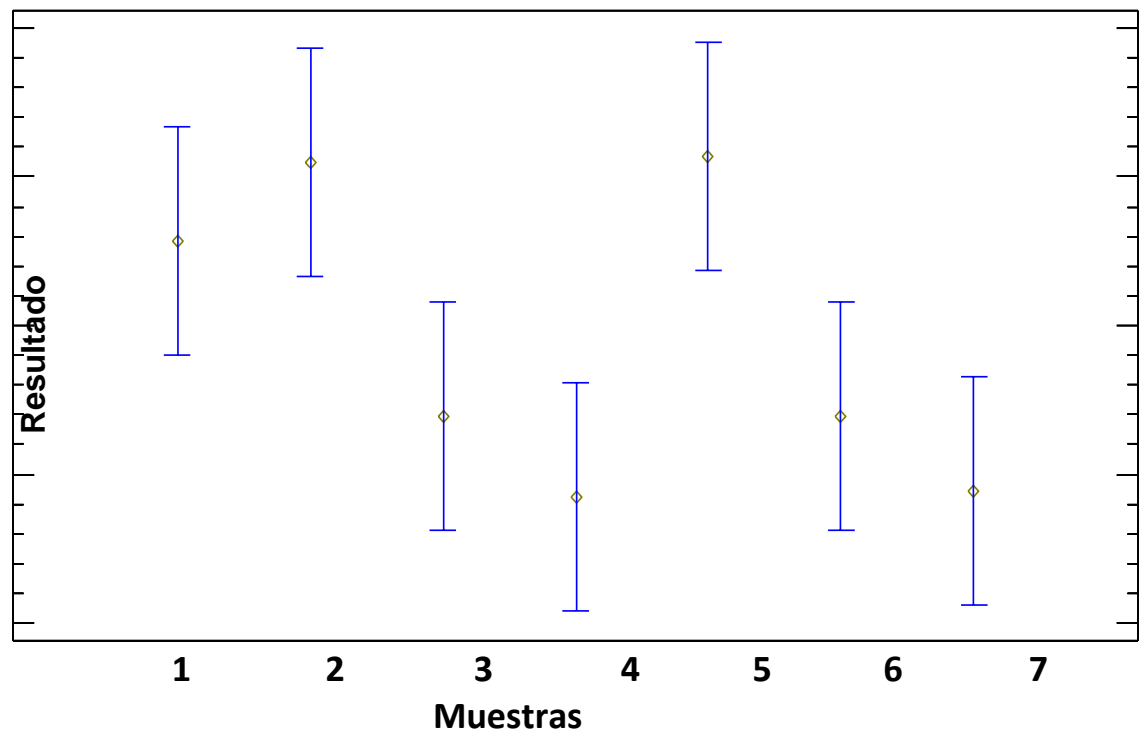

\section{Discusión}

La aceptabilidad de las muestras de anisado elaboradas mediante redestilación de fracciones de cabeza de destilado de pisco de uva Negra Criolla, tuvo como resultado en el mejor de los casos una aceptación de 6,54 en una escala hedónica de 1 a 9. Caso similar al obtenido por Williams y Strauss (1976) quienes, mediante un procedimiento para eliminar el acetaldehído de las fracciones de cabeza de destilación de vino de uva, que implicó el burbujeo con gas inerte y reflujo, obtuvo un valor de entre 5,1 a 8,3, para las fracciones de cabeza tratadas, concluyendo que es posible obtener aguardientes de calidad similar a los aguardientes comerciales de alta calidad. Considerando además que el proceso de redestilación practicado, también implica la separación de una nueva fracción de cabeza, que como explica Hatta (2004), en la figura 3, sobre la evolución de los componentes volátiles del pisco durante la destilación, la fracción de acetaldehídos se obtiene mayoritariamente en la primera quinta parte del proceso de destilación.

Respecto a la aceptabilidad del anisado elaborado mediante redestilación de fracciones de cabeza de destilado de pisco de uva Negra Criolla, se debe precisarel posible temor al metanol, ya que mientras que Williams (1976), indicaba que las fracciones de cabeza contienen al menos $80 \%$ de etanol, Hatta (2004), que han investigado la porción de etanol en cada fase de la destilación, muestra una distribución casi uniforme e incluso mayor durante la destilación del cuerpo, por lo que existe una contradicción entre ambos planteamientos.

\section{Conclusión}

Se aprovechó las fracciones de cabeza del destilado de pisco de uva Negra Criolla mediante redestilación para la elaboración de anisado.

Se analizó las fracciones de cabeza de pisco de uva Negra Criolla, presentando un grado alcohólico de $61^{\circ} \mathrm{GL}$ y una densidad de 1,5228 .

Se logró elaborar las muestras de anisado por el método de destilación directa de las fracciones de cabeza de la destilación del pisco de uva Negra Criolla, con los granos de anís (Pimpinella anissum), 
logrando siete muestras las mismas que se lograron analizar fisicoquímicamente presentando una relación estadísticamente significativa entre la densidad y el volumen, densidad y rendimiento, así como densidad y análisis sensorial del anisado, con un nivel de confianza del 95,0\%. Así mismo existe una relación estadísticamente significativa entre el rendimiento y el análisis sensorial.

Se evaluó la aceptabilidad de las muestras de anisado elaboradas con fracciones de cabeza de destilado de pisco de uva Negra Criolla, siendo valoradas en el programa estadístico statgraphics obteniéndose que los valores-P prueban la significancia estadística entre la aceptabilidad y las muestras con valor-P menor que 0,05 , este factor tiene un efecto estadísticamente significativo sobre las muestras con un 95,0 \% de nivel de confianza, resultando la muestra5 con proporción 20:79:01 (cabeza, agua y anís), con una aceptaciónde 6,54 en una escala hedónica de 1 a 9.

Realizar un estudio comparativo de las concentraciones de anetol enanisados con otros tipos de plantas.

Optimizar las proporciones de los componentes del anisado a partirde las fracciones de cabeza de destilado de pisco.

Determinar los factores que provocan las alteraciones en el color delos destilados anisados.

Establecer un léxico para los análisis sensoriales del anisado a partirde los productos destilados.

\section{Referencias Bibliográficas}

Albert, P. C. (1999). Productos de Calidad: Los anises de Cazalla y de Rute. Agricultura: Revista agropecuaria, 644-646.

Alberto Garrido S., T. L. (2008). Estudio de la composición de las fracciones de destilado en un proceso de obtención de pisco. Revista Peruana Química e Ingeniería Química, 19-22.

Camacho, A. (2009). http://depa.fquim.unam.mx/amyd/archivero. Recuperado el 2de 12 de 2015, de http://depa.fquim.unam.mx/amyd/archivero:

http://depa.fquim.unam.mx/amyd/archivero/TecnicBasicas-Colif-tot-fecales- EcoliNMP_6529.pdf

Chen, J. C. (1991). Manual del azúcar de caña. México: Limusa.

Dambergs, R. G. (2002). Rapid analysis of methanol in grape-derived distillation products using nearinfrared transmission spectroscopy. Journal of agricultural and food chemistry, 3079-3084.

Diccionario de la Lengua Española. (2016). Real Academia Española. Recuperado el 20 de Enero de 2016, de http://dle.rae.es/: http://lema.rae.es/drae/srv/search?key=rendimiento

Fitz, A. R. (2006). Desarrollo de un Experimento de Destilación para Mezclas Binarias a nivel Planta Piloto. México.

Geankoplis, C. J. (2006). Procesos de transporte y principios de procesos deseparación: incluye operaciones unitarias. México DF: Patria.

Gobierno Regional de Tacna. (2009). Plan Estratégico Regional del Sector Agrariode Tacna 2008 2015. Tacna: GORE-TACNA.

Hatta, B. (2004). Influencia de la fermentación con orujos en los componentes volátiles del pisco de uva italia (Vitis vinifera L. var. Italia). Lima.

Holanda, C. D. (1981). Fundamentos de la destilación multicomponente. Nueva York: McGraw-Hill.

INDECOPI. (11 de diciembre de 2014). Norma Técnica Peruana NTP211.015. Bebidas alcohólicas. Anis o anisado. Requisitos. Lima, Lima, Perú: INDECOPI. 
INDECOPI, C. d. (02 de Noviembre de 2006). NTP 211.001. NORMA TÉCNICAPERUANA. Lima, Lima, Perú: INDECOPI.

Jurado J., J. M. (2004). Caracterización analítica de aguardientes anisados. Sevilla:Universidad de Sevilla.

Jurado, J.M. (2006). LC determination of anethole in aniseed drinks. Chromatographia, 223-226.

Karapanagioti, H. K. (2014). Alcohol and Dilution Water Characteristics in Distilled Anis (Ouzo). Journal of agricultural and food chemistry, 4932-4937.

López V., C. (2011). Estudio del comportamiento de columnas de destilación en la elaboración de aguardientes de orujo. Santiago de Compostela: Universidadde Santiago de Compostela.

Olguín Fonseca, F. G. (2012). Elaboración de alcohol rectificado a partir de la melaza en la Empresa Agroindustrial Paramonga SSA periodo 2011. Huacho: Universidad Nacional José Faustino Sanchez Carrión.

Omidbaigi, R. H. (2003). Changes in content and chemical composition of Pimpinella anisum oil at various harvest time. Journal of Essential Oil Bearing Plants, 6(1), 46-50.

Pino, O. S. (2012). Composición química y actividad antibacteriana del aceite esencial de Pimpinella anisum L. Revista de Protección Vegetal, 181-187.

Rakoff, H. \&. (1992). Química Orgánica Fundamental. México D. F., México, México: LIMUSA, S. A.

Ribéreau-Gayon, P. -D.-D.-L. (2003). Tratado de Enología - Microbiología del vino - Vinificaciones (Vol. I Volumen). (A. Dayan, Trad.) Dunod, París, Francia: Mundi-Prensa Libros S. A.

Ribéreau-Gayon, P. -G.-M.-D. (2002). Tratado de Enología - Química del vino - Estabilización y tratamientos (Vol. II). (M. T. Miccio, Trad.) París, Dunod, Francia: Ediciones Mundi-Prensa.

Rota, M. B. (2009). Efeito do processo de bidestilacao na qualidade sensorial da cachaca. Araraquara - Brasil: UNESP - Facultad de Ciencias Farmacéuticas.

Tsachaki, M. A. (2010). Development of a suitable lexicon for sensory studies of the anise-flavoured spirits ouzo and tsipouro. Flavour and fragrance journal, 25(6), 468-474.

Williams, P. J. (1976). A treatment of grape wine distillation heads. Journal of the Science of Food and Agriculture, 487-498.

Yilmaztekin, M. C. (2011). Differentiation of Turkish Rakies through Headspace Solid-Phase Microextraction and Gas Chromatography-Mass Spectrometry Analysis. Journal of the Institute of Brewing, 117(4), 622-626. 